\title{
SENSITIVITY OF THE TUBERCLE BACILLUS TO STREPTOMYCIN BEFORE AND DURING SPECIFIC THERAPY ${ }^{1,2,3}$
}

\author{
By JOSEPH F. SADUSK, JR., AND WILLIAM E. SWIFT, JR. \\ With THE technical assistance of ELEANORA FALCO AND JOHN VADNEY \\ (From the Department of Internal Medicine, Yale University School of Medicine, New Haven, \\ and the Laurel Heights Sanatorium, Shelton, Conn.)
}

(Received for publication December 15, 1947)

Shortly after the demonstration of a therapeutic effect of streptomycin upon tuberculous infections in animals $(1,2)$ and in the human (3), Youmans and his co-workers (4) found that cultures from patients subsequent to such specific therapy yielded organisms which were resistant to streptomycin. These investigators not only observed that 7 of 8 strains recovered from 12 patients before and after treatment with streptomycin exhibited an increase in resistance of from 500 - to 1,000-fold compared to tests performed prior to initiation of therapy, but they also showed that the resistance of two strains of Mycobacterium tuberculosis, one of the human type and one of the avian type, increased more than 1,000fold when the organisms were exposed to streptomycin in vitro.

The development of such resistance, or perhaps better expressed as loss of sensitivity, of the tubercle bacillus during streptomycin therapy has recently been studied in a joint program of the Yale University School of Medicine and the Laurel Heights Sanatorium, carried out under the auspices of the American Trudeau Society. The results of this study are presented below.

- Presented in part at a symposium on streptomycin therapy of pulmonary tuberculosis before the Yale Medical Society, New Haven, May 14, 1947; and at the Eastern Sectional Meeting of the American Federation for Clinical Research, New York City, December 12, 1947.

2 This study was aided by a grant from the Fluid Research Fund of the Yale University School of Medicine.

8 This study is part of the Streptomycin-Tuberculosis Research Project of the American Trudeau Society, Medical Section, National Tuberculosis Association. The drug was generously donated to the Society by the following: Abbott Laboratories, Eli Lilly \& Co., Merck \& Co., Inc., Charles Pfizer \& Co., Inc., E. R. Squibb \& Sons, and the Upjohn Co. Collateral clinical observations were made at the Laurel Heights Sanatorium by Dr. Kirby S. Howlett, Jr., and Dr. John B. O'Connor to whom the authors are indebted for generous advice and assistance with the reported study.

\section{METHODS}

A group of 16 patients with pulmonary tuberculosis was treated with 1.8 grams per day of streptomycin in 6 divided doses until late in the course of treatment when the same daily amount of streptomycin was divided into 5 doses, omitting the 3:00 a.m. dose. In most instances, treatment was discontinued after four months. Eight of these patients also had ulcerative tuberculous bronchitis.

Sputa or gastric washings were collected at routine intervals. Tubercle bacilli were isolated from these specimens by inoculation of the neutralized alkaline concentrate on Hohn's and egg yolk media. After satisfactory growth had been obtained, transfer of part of a suitable colony was made to a tube of modified Dubos-Davis medium $(5,6)$ with a nichrome. spatula, mashing and triturating the colony in the medium in order to give as diffuse a suspension as possible. Later on in the work it was found desirable routinely to add penicillin (20 units per cc.) to the Dubos medium used for first transfer from the solid slants in order to prevent contamination with gram positive cocci. After a growth period of from 7 to 10 days at $37^{\circ} \mathrm{C}$., tubercle bacilli grew in a lacey pattern at the bottom of the tube in this initial transfer. In subsequent transfers, such growth appeared as early as the third or fourth day. When growth was sufficient after shaking to cause turbidity approximately equal to that of the No. $2 \mathrm{McF}$ arland nephelometer scale, the culture tube was well shaken and allowed to stand for 15 minutes to allow larger particles to settle out. Pyrex tubes, each containing $5.0 \mathrm{cc}$. of the Dubos-Davis medium to which had been added the requisite quantity of streptomycin, were then inoculated with $0.1 \mathrm{cc}$. of the turbid supernatant culture. The following concentrations of streptomycin were routinely employed: $0.5,1.0,5.0,10.0$, $50.0,100.0,500.0$, and $1,000.0 \mathrm{mcg}$. per cc. In several instances, concentrations of $2.0,3.0$, and $4.0 \mathrm{mcg}$. per cc. were also used. Daily readings were made from the fourth to the 12th days, the end point being taken as the tube containing the lowest concentration of streptomycin which was sufficient to inhibit the growth of tubercle bacilli. Ordinarily, no change was observed after the 7th day of incubation. Growth of tubercle bacilli was confirmed by acid fast stain of the culture.

Pyrex tubes used in the experiment were $25 \times 150 \mathrm{~mm}$. in size. Precautions concerning chemical cleanliness of these tubes and the preparation of cotton plugs as outlined by Dubos and Davis (5) were carefully observed. 
Details of the preparation of the modified Dubos-Davis medium are given below:

\section{Mineral Concentrate}

Sodium phosphate, $\mathrm{Na}_{2} \mathrm{HPO}_{4} \cdot 12 \mathrm{H}_{2} \mathrm{O}-12.5 \mathrm{grams}$

Potassium phosphate, $\mathrm{KH}_{2} \mathrm{PO}_{4}-2.0$ grams

Sodium citrate, $\mathrm{Na}_{3}$ citrate $2 \mathrm{H}_{2} \mathrm{O}$ - 3.0 grams

Magnesium sulfate, $\mathrm{MgSO}_{4} 7 \mathrm{H}_{2} \mathrm{O}-1.2$ grams

Distilled water Adjust to $\mathrm{pH} 7.2$

2. Stock Medium

Mineral concentrate (No. 1)

$\mathrm{N}-\mathrm{Z}$ Amine, ${ }^{4} 5 \%$ in dist. water

Vegex, $5 \%$ in dist. water

Ferric ammonium citrate

Asparagine

Distilled water

$-100.0 \mathrm{cc}$.

$-100.0 \mathrm{cc}$.

$-40.0 \mathrm{cc}$.

- $4.0 \mathrm{cc}$.

- $0.10 \mathrm{gram}$

- 2.0 grams

$-800.0 \mathrm{cc}$.

Store in stoppered flask in ice box, adding about $5 \mathrm{cc}$. of chloroform as "puddle" in bottom of flask. This solution may be thus stored for several months.

3. Tween 80 (lot 1405), ${ }^{6} \cdot 10 \%$ in dist. water. Keep in ice box for not longer than 1 week before using.

4. Dextrose, $25 \%$ in dist. water, autoclaved at $15 \mathrm{lb}$. for 30 minutes.

5. Albumen $75 \%$, bovine plasma fraction $\mathrm{V}$ in $0.85 \%$ sodium chloride. Neutralize to $\mathrm{pH} 7.0$ with sodium hydroxide, filter through Seitz filter under aseptic conditions, heat to $55^{\circ} \mathrm{C}$. for 30 minutes in water bath in order to inactivate lipase.

6. Final Medium

Add 0.2 cc. of $10 \%$ Tween 80 solution (No. 3) to $100 \mathrm{cc}$. of stock medium (No. 2). Autoclave at $15 \mathrm{lb}$. for 20 minutes; when cool, add $2.0 \mathrm{cc}$. of $25 \%$ dextrose solution (No. 4) and $10.0 \mathrm{cc}$. of $5 \%$ albumen solution (No. 5). Dispense $5.0 \mathrm{cc}$. each in pyrex tubes $(25 \times 150$ $\mathrm{mm}$.) with requisite concentrations of streptomycin.

\section{RESULTS}

Strains of tubercle bacilli recovered from the group of 16 patients suffering from pulmonary tuberculosis and treated with streptomycin were tested for their sensitivity to the drug in the manner described above. The results are presented in Table I.

All 16 strains of the tubercle bacillus recovered prior to treatment were highly susceptible to the in vitro action of streptomycin. Fourteen of these strains were inhibited by a concentration of

"Sheffield Farms Co., 524 West 57th Street, New York City.

5 Vegex Company, 175 Fifth Avenue, New York City.

- "Tween 80 " is the trade name of a polyoxyethylene derivative of sorbitan monooleate. It was furnished through the courtesy of the Atlas Powder Company, Wilmington, Delaware.

7 Armour Laboratories, Chicago, Illinois.
TABLE I

\begin{tabular}{|c|c|c|c|c|c|}
\hline Patient & $\begin{array}{l}\text { Pre- } \\
\text { treat- } \\
\text { ment }\end{array}$ & $\begin{array}{l}\text { End of } \\
\text { 1st } \\
\text { month }\end{array}$ & $\begin{array}{l}\text { End of } \\
\text { 2nd } \\
\text { month }\end{array}$ & $\begin{array}{l}\text { End of } \\
\text { 3rd } \\
\text { month }\end{array}$ & $\begin{array}{l}\text { End of } \\
\text { 4th } \\
\text { month }\end{array}$ \\
\hline $1-F h$ & $m c g . / c c$. & mcg./cc. & mcg. $/ c c$. & $m c g . / c c$ & mcg./cc. \\
\hline ase $2-\mathrm{Ge}$ & 0.5 & 5.0 & 50.0 & 0.5 & 10.0 \\
\hline Case $3-\mathrm{Gi}$ & 0.5 & & 10.0 & & \\
\hline Case $4-K o$ & 0.5 & 5.0 & 3.0 & 5.0 & 5.0 \\
\hline Case 5-Ma & 0.5 & 0.5 & 5.0 & $>1000.0$ & \\
\hline Case $6-\mathrm{Mi}$ & 0.5 & 0.5 & 0.5 & 5.0 & 5.0 \\
\hline Case $7-\mathrm{Ob}$ & 0.5 & 0.5 & & & \\
\hline Case $8-\operatorname{Re}$ & 0.5 & 0.5 & & & 10.0 \\
\hline Case 9-Ro & 0.5 & 0.5 & & & \\
\hline $\begin{array}{l}\text { Case } 10-\text { Si } \\
\text { Case } 11-\text { So }\end{array}$ & 0.5 & 1.0 & $\begin{array}{r}500.0 \\
0.5\end{array}$ & 500.0 & . \\
\hline $\begin{array}{l}\text { ase } 11-\mathrm{So} \\
\text { ase } 12-\mathrm{Su}\end{array}$ & $\begin{array}{l}1.0 \\
0.5\end{array}$ & $\begin{array}{l}0.5 \\
0.5\end{array}$ & 0.5 & & \\
\hline ase $13-$ Vel & 0.5 & 0.5 & 0.5 & & \\
\hline ase $14-$ Ver & 1.0 & 0.5 & 0.5 & & 50.0 \\
\hline ase $15-\mathrm{Wa}$ & 0.5 & 0.5 & & 50.0 & \\
\hline ase $16-\mathrm{Wi}$ & 0.5 & 5.0 & 4.0 & $>1000.0$ & $>1000.0$ \\
\hline
\end{tabular}

$0.5 \mathrm{mcg}$. of streptomycin per cc.; the remaining two strains were inhibited by $1.0 \mathrm{mcg}$. of the agent per cc.

At the end of the first month of therapy, isolation of the organism by culture was successful in 14 of the cases. Except for three instances where there was a ten-fold increase in resistance to streptomycin, there was no significant change in reaction to the in vitro effect of streptomycin of the remaining strains.

By the end of the second month of therapy, not only did attempts at isolation of the tubercle bacilli from sputa or gastric contents reveal an increasing number of negative specimens, but also an increasing frequency and degree of resistance of the organisms to the chemotherapeutic agent. For instance, negative cultures had increased from 2 after the first month of therapy to 5 by the end of the second month. Along with this, 4 of the 11 isolated strains now showed a ten-fold or greater. increase in resistance. In 2 of these 4 resistant strains, resistance had increased 100 -fold and 1000-fold, respectively.

During the third and fourth months of therapy the number of negative specimens and resistance of those organisms obtained by culture continued to increase. At the end of the third month, positive cultures were obtained in 7 of the 16 cases. In all but one instance, a ten-fold or greater in- 
crease in resistance was demonstrated. Two of the 6 resistant strains showed over a 2,000-fold increase, while tests on the remaining 4 of these 6 strains indicated that resistance had increased ten-fold in two instances, and 100-fold and 1,000fold in the remaining two, respectively.

By the end of the course of therapy at the fourth month, 6 positive cultures were obtained. Tests of these organisms showed a ten-fold increase in resistance in two cases, 20 -fold in two cases, 50 -fold in one case and over 2,000-fold in the remaining case.

\section{DISCUSSION}

The results described above show a general tendency toward a steady reduction in the number of culturally positive sputa and gastric contents while the patients were under treatment with streptomycin. They further demonstrate the progressive development of significant streptomycin resistance of remaining organisms during such specific therapy. It should be stated at this point that the term "development of resistance" is loosely used throughout this report; the authors do not imply that a given genetic strain actually "develops" resistance but merely that resistant organisms appear. A discussion of whether this "resistance" depends upon the selection of gehetically resistant substrains in a culture, as has been shown with other micro-organisms and other chemotherapeutic agents, or whether true resistance of a given genetic strain of the Mycobacterium tuberculosis actually develops during exposure to streptomycin is beyond the scope of the present communication. Pyle (7) has very recently brought forth evidence which indicates that the former suggestion is correct.

It is of considerable interest that all organisms isolated after the third or fourth month of streptomycin therapy in the present study showed at least a ten-fold increase in resistance. This holds for 9 of the 16 cases investigated. In the remaining 7 cases, cultures had reverted to negative by this period.

Breakdown of the 9 strains of organisms isolated after the third or fourth month of therapy indicates the following degree of development of resistance when compared to the sensitivity of the strain isolated prior to treatment: 10-fold increase in resistance-2

20 -fold increase in resistance-2

50 -fold increase in resistance-1

100 -fold increase in resistance- 1

1,000 -fold increase in resistance-1

2,000-fold increase in resistance-2

Total number of strains isolated at end of therapy $-9$

The clinical significance of this development of resistance is not clear at the present time. Indeed, the exact level at which organisms may be called "resistant" has not even been defined. While there is no conclusive clinical evidence in the human to indicate that in vivo and in vitro resistance parallel each other, Youmans and Williston (8) have shown that tuberculous infections produced in mice with streptomycin-resistant cultures of the tubercle bacillus were not suppressed by treatment of the animals with streptomycin.

Although it is not possible to attempt any definite correlation between the development of resistant organisms and the clinical course of the patients treated in the present study the following general statements, particularly with reference to the four cases in which resistance had increased at least 100 -fold, may be made (9):

1. Definite relapse (clinical, radiologic, and bronchoscopic) occurred under treatment in one patient at about the time that the tested organisms showed a 2,000-fold increase in resistance.

2. Of the other three cases showing highly resistant strains during treatment, two continued to improve satisfactorily; the other case never showed significant improvement at any time.

3. One patient who relapsed under treatment (fever, increased expectoration, recurrence of grossly positive sputum, but no unfavorable $\mathrm{x}$-ray change) and another patient who failed to maintain peak improvement, each yielded a strain of organism showing only a ten-fold increase in resistance.

It is evident from the foregoing data that it is impossible at this time to draw any definite correlation between in vitro resistance of the causative organism and the therapeutic course in the small number of cases treated. Further observations with respect to this correlation (9) and data concerning the resistance of organisms to strep- 
tomycin in the post-treatment period (10) will be recorded at a later date.

While investigators agree that tubercle bacilli develop a variable degree of resistance to streptomycin during therapy, they are not all in harmony as to the number which do so. Part of the disagreement, at least, may be due to two factors: first, definition of the term "resistance," and second, use of different technical procedures and media for the assay of resistance. Youmans et al. (4) first reported the development of resistance; they found that tubercle bacilli isolated from 8 of 12 patients after treatment with streptomycin showed a marked resistance to streptomycin. Resistance of 7 of these 8 strains, when compared with the resistance of strains isolated from the same patients before treatment, had increased 500 - to 1,000 -fold. These findings have been very recently confirmed by Youmans and Karlson (11). McDermott's observations (6) indicate that virtually all strains isolated after the 12th week of therapy gave sensitivity values of $100 \mathrm{mcg}$. or higher (equivalent to an increase in resistance of approximately 200 -fold or higher). In some instances this resistance developed as early as the end of the first month of therapy. Studies carried out under the auspices of the Veterans Administration (12) show that $78 \%$ of positive cultures obtained from streptomycin-treated patients at the end of 120 days contained organisms which were resistant to more than $10 \mathrm{mcg}$. of streptomycin per cc. (20-fold increase).

In striking contrast to the above observations which are in accord with the findings in the presently reported paper, Wallace and Fisher (13) found that only 4 of 20 strains (20\%), isolated after four months of streptomycin therapy, manifested resistance to the drug when tested in Dubos medium. Resistance was defined as growth in 10 mcg. or more streptomycin per cc. of medium. Utilizing Youman's medium (11) as the test medium for determination of sensitivity of these same tubercle bacilli to streptomycin, Wallace and Fisher now found that 11 of the 20 strains (55\%) could be classed as resistant, using the definition as given above.

It is possible that the low incidence of appearance of streptomycin-resistant strains reported by Wallace and Fisher with Dubos medium may be due to the use of a medium with a relatively low concentration of albumin and a relatively high concentration of Tween 80 , thus giving falsely low values for the streptomycin sensitivity of tubercle bacilli. In our experiments, albumen concentrations of $0.5 \%$ and Tween 80 concentrations of $0.02 \%$ in the final medium were employed, thus duplicating the conditions advised by McDermott and his collaborators (6) who have particularly stressed the necessity of using a low concentration of Tween 80 since this agent appears to possess a bacteriostatic action upon the growth of the tubercle bacillus.

In view of the disagreement noted above, it is also natural to inquire as to the possibility of selection of colonies of varying degrees of resistance to streptomycin from the same culture, a phenomenon previously described with non-acid fast organisms. In a number of instances, attempts were made to check the sensitivities of some of the strains of tubercle bacilli described in Table I, utilizing the same culture but different colonies. In some instances cultures isolated within a week or two of the reported strains were also employed. The results show that while the values obtained were in general agreement (within limits of technical error) with the values previously obtained, there were notable exceptions not only to the specific sensitivity value for a given date but also to the general trend. This point obviously needs further clarification since the authors are satisfied with the relative accuracy of the procedure outlined for determination of sensitivity.

\section{SUMMARY}

In vitro streptomycin sensitivity of tubercle bacilli isolated from sputa or gastric contents was determined in a group of 16 patients with pulmonary tuberculosis receiving 1.8 grams of streptomycin daily for a period of four months. In vitro tests were performed in a modified Dubos-Davis medium.

In all 16 cases, the strains of bacilli isolated prior to treatment were highly sensitive to streptomycin. Fourteen of these 16 strains were inhibited by $0.5 \mathrm{mcg}$. of streptomycin per cc., the remaining 2 strains were inhibited by $1.0 \mathrm{mcg}$. per cc.

Loss of sensitivity (ten-fold increase in resistance) began to appear by the end of the first month of therapy together with conversion of 
positive sputum or gastric washings to negative in other cases as determined by culture.

By the end of the third and fourth months of therapy, cultures were positive in only 9 of the 16 cases. Sensitivity tests of organisms from these 9 positive cultures indicated that all of them had developed a ten-fold or greater increase in resistance. Five of these 9 strains developed a 10to 50-fold increase in resistance; the remaining 4 strains developed a 100 to greater than 2,000 fold increase in resistance.

It was not possible with this small group of patients to demonstrate conclusively a correlation between resistance to streptomycin and clinical course under therapy.

\section{BIBLIOGRAPHY}

1. Feldman, W. H., and Hinshaw, H. C., Effects of streptomycin on experimental tuberculosis in guinea pigs: A preliminary report. Proc. Staff Meet., Mayo Clinic, 1944, 19, 593.

2. Feldman, W. H., and Hinshaw, H. C., Streptothricin in experimental tuberculosis. Am. Rev. Tuberc., 1945, 52, 299.

3. Hinshaw, H. C., Feldman, W. H., and Pfuetze, K. H., Streptomycin in treatment of clinical tuberculosis. Am. Rev. Tuberc., 1946, 54, 191.

4. Youmans, G. P., Williston, M. A., Feldman, W. H., and Hinshaw, H. C., Increase in resistance of tubercle bacilli to streptomycin: A preliminary report. Proc. Staff Meet., Mayo Clinic, 1946, 21, 126.

5. Dubos, R. J., and Davis, B. D., Factors affecting the growth of tubercle bacilli in liquid media. J. Exper. Med., 1946, 83, 409. 6a. McDermott, W., Muschenheim, C., Hadley, S. J., Bunn, P. A., and Gorman, R. V., Streptomycin in the treatment of tuberculosis in humans. I. Meningitis and generalized hematogenous tuberculosis. Ann. Int. Med., in press.

b. Muschenheim, C., McDermott, W., Hadley, S. J., Hull-Smith, H., and Tracy, A., Streptomycin in the treatment of tuberculosis in humans. II. Pulmonary tuberculosis. Ann. Int. Med., in press.

7. Pyle, M. M., Relative numbers of resistant tubercle bacilli in sputa of patients before and during treatment with streptomycin. Proc. Staff Meet., Mayo Clinic, 1947, 22, 465.

8. Youmans, G. P., and Williston, E. H., Effect of streptomycin on experimental infections produced in mice with streptomycin resistant strains of $M$. tuberculosis var. Hominis. Proc. Soc. Exper. Biol. \& Med., 1946, 63, 131.

9. Howlett, K. S., Jr., and O'Connor, J. B., to be published.

10. Swift, W. E., Jr., and Beardsley, F. A., Jr., to be published.

11. Youmans, G. P., and Karlson, A. G., Streptomycin sensitivity of tubercle bacilli; studies on recently isolated tubercle bacilli and the development of resistance to streptomycin in vivo. Am. Rev. Tuberc., 1947, 55, 529.

12. Veterans Administration Technical Bulletin, T.B. 1037, dated September 24, 1947, The effect of streptomycin upon pulmonary tuberculosis in man-Preliminary report of a cooperative study of 223 cases by the Army, Navy, and Veterans Administration. Also published as Effects of streptomycin on tuberculosis in man, J. A. M. A., 1947, 135, 634.

13. Wallace, J. B., and Fisher, M. W., unpublished data, Study Unit Reports, March 1947, St. Louis Streptomycin Conference, May 1-3, 1947, Veterans Administration Streptomycin Committee, Central Office, Washington, D. C. 